\title{
Ansatz zur belastungsgerechten Auslegung additiv gefertigter Meso-Strukturen in Bauteilen
}

\author{
Fiona Schulte ${ }^{1 *}$, Eckhard Kirchner ${ }^{1}$ \\ 1 Technische Universität Darmstadt \\ * Korrespondierender Autor: \\ Fiona Schulte \\ TU Darmstadt \\ Institute of Product Development and Machine Elements \\ Otto-Berndt-Straße 2, 64287 Darmstadt \\ Telefon: 06151/16-21208 \\ Mail: schulte@pmd.tu-darmstadt.de
}

\begin{abstract}
Additive manufacturing enables the production of defined cellular and lattice structures. These additively manufactured meso structures offer various potentials like lightweight design or defined inner properties. To reduce the design effort a methodological approach is derived to enable fit for purpose design of components with meso structures using analytical calculation rules. The analogy to the analytical calculation of solid components emphasises the relevant parameters for the design as well as their influence on the properties of meso structures. Furthermore, based on the streamlined preliminary design, indications for the selection of suitable elementary cells regarding arising, even inhomogeneous, stresses are provided. The design method is exemplary applied to a cantilever beam.
\end{abstract}

\footnotetext{
Keywords

additive manufacturing, meso structures, lattice structures, systematic design, fit for purpose design, preliminary design, equivalent properties
} 


\section{Motivation}

Die additive Fertigung (AM) bietet durch ihren schichtweisen Bauteilaufbau eine hohe geometrische Freiheit und u. a. die Möglichkeit, filigrane Strukturen und Hohlräume oder Hinterschnitte herzustellen. Dadurch können sowohl offene Leichtbau-Tragwerke als auch Leichtbauvolumenbauteile hergestellt werden. Leichtbauvolumenbauteile zeichnen sich dadurch aus, dass eine "massive" Wand die Körperkontur bildet und das Material im Bauraum im Inneren lokal und entsprechend der Beanspruchung angeordnet wird z. B. durch den Einsatz von Topologieoptimierungen oder definierten Zell- bzw. Gitterstrukturen. Die Größe der Gitterstrukturen liegt im Bereich der Meso-Skala, ca. 0,1 bis $10 \mathrm{~mm}$ [1], daher werden sie hier als Meso-Strukturen bezeichnet. Die additiv gefertigten Meso-Strukturen bieten diverse Potentiale, z. B. Leichtbau oder die Realisierung definierter innerer Eigenschaften in Bauteilen [2, 3], die von großem Interesse für die Bauteilentwicklung sind, da sie eine Optimierung von Bauteilen hinsichtlich Gewicht und damit $u$. a. Kosten, aber auch hinsichtlich zugeschnittener Eigenschaften darstellen. Durch unterschiedliche Gitterzellen, die bereits entwickelt und untersucht worden sind, können eine Vielzahl verschiedener Eigenschaften realisiert werden. Um diese Potentiale zielgerichtet nutzen zu können, ist es wichtig, sie den Entwicklern systematisch und strukturiert zugänglich zu machen. Ein wichtiger Aspekt der systematischen Entwicklung mit additiv gefertigten Meso-Strukturen ist ihre Auslegung, die ein Abstimmen der Eigenschaften von Meso-Strukturen auf gestellte Anforderungen ermöglicht.

\section{Grundlagen}

Die Möglichkeit Meso-Strukturen mittels AM herzustellen und anzuwenden, wird in den letzten Jahrzehnten zunehmend untersucht. Dabei unterscheiden sich die AM-MesoStrukturen von Schäumen, deren Größe ebenfalls im Bereich der Meso-Skala liegt, durch die definierte Geometrie ihrer Elementarzellen.

Im Bereich AM wurde bereits eine Vielzahl von Meso-Strukturen entwickelt und untersucht, die sich in drei Elementarzelltypen unterteilen lassen, Elementarzellen aus Stäben (Stabgitter), ebenen Platten (Plattengitter) [4] und Schalen (Schalengitter). Deren Eigenschaften werden experimentell oder simulativ häufig in Druckversuchen untersucht. Bei Untersuchungen zu Anwendungen von Meso-Strukturen werden meist ausgewählte Elementarzellen hinsichtlich ihrer Eignung für die Erfordernisse der Anwendung verglichen u. a. [5-7]. Dabei wird deutlich, dass für die Entwicklung eines Bauteils mit AM-Meso-Strukturen zunächst eine geeignete Elementarzelle gewählt und diese anschließend dimensioniert und auf die Beanspruchungen im Bauteil abgestimmt werden muss. Für die Auswahl ist die Kenntnis der Eigenschaften, insbesondere Festigkeits- und Steifigkeitseigenschaften, der Elementarzellen erforderlich.

Zur Beschreibung der Eigenschaften wird in der Literatur der Ansatz der äquivalenten Kontinuumseigenschaften beschrieben [5-7]. Dabei wird die betrachtete Elementarzelle einzeln mittels Finite-Elemente-Methode (FEM) simuliert und deren Eigenschaften, z. B. die Steifigkeit, häufig mit dem äquivalenten Elastizitätsmodul $\mathrm{E}^{*}$ bezeichnet, in Abhängigkeit vom Füllgrad $\rho^{*}$ (auch als relative Dichte bezeichnet) aus den Simulationsergebnissen ermittelt. Diese ermittelte Steifigkeit wird dann dem Volumen des meso-strukturierten Bauteils oder Bauteilbereichs für die simulative Berechnung des Bauteils zugewiesen, wobei die Annahme zugrunde liegt, dass das meso-strukturierte Volumen analog zum homogenen Material als Kontinuum mit den äquivalenten Kenngrößen $\rho^{*}$ und $E^{*}$ betrachtet werden kann. Dadurch wird insbesondere die Simulation mittels FEM gegenüber der Simulation der tatsächlichen MesoStruktur-Elementarzellen signifikant vereinfacht und beschleunigt und die Ergebnisse zeigen eine gute Übereinstimmung mit der Simulation ohne Vereinfachung sowie experimentellen Ergebnissen für den jeweiligen Belastungsfall [5-7]. Bei der Anwendung der äquivalenten Kontinuumseigenschaften werden die Größen für jede Elementarzelle aus der Simulation 
ermittelt. Dabei wurden bisher die Eigenschaften ausgewählter würfelförmiger orthotroper Elementarzellen ermittelt u. a. [5-8].

\section{Forschungsfrage}

Der Ansatz der äquivalenten Kontinuumseigenschaften vereinfacht die simulative Berechnung meso-strukturierter Bauteile, allerdings erfolgt die Dimensionierung der Elementarzellen über Iterationen der Simulation, um die Meso-Struktur auf die Beanspruchung im Bauteil auszulegen. Dabei weisen die ausgewählten Elementarzellen nicht zwingend eine beanspruchungsgerechte Geometrie auf. Oft werden homogen verteilte und dimensionierte, d. h. Elementarzellen mit gleicher Form, Größe, Topologie und Orientierung, orthotrope oder isotrope Elementarzellen betrachtet [5], während die Beanspruchung im Bauteil in vielen Fällen anisotrop und inhomogen ist und zwischen Bauteilbereichen in Intensität, Art oder Richtung variiert. Dies wird am einfachen Beispiel eines Kragträgers unter Querkraft in Abbildung 1 ersichtlich. In den Außenbereichen überwiegen Zug- bzw. Druckspannungen, die in Richtung der Einspannstelle zunehmen, während im Bereich der neutralen Faser des Kragträgers weitgehend konstante Schubspannungen dominieren. Während Leichtbaustrukturen, die sich über das gesamte Bauteil erstrecken, z. B. ausgehend von der Theorie der Spannungstrajektorien, mittels analytischer Gleichungen ausgelegt werden können, wird die Auslegung inhomogener und anisotroper Meso-Strukturen auf ähnliche Weise derzeit nicht unterstützt (vgl. dazu auch Abbildung 1b und [9]).

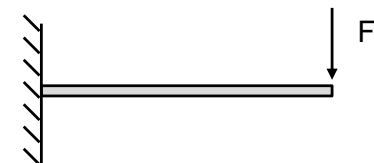

a) Kragträger

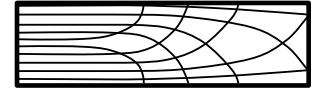

b) Spannungstrajektorien

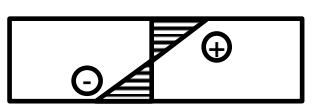

c) Zug-Druckspannungsverteilung

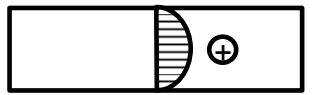

d) Schubspannungsverteilung

Abbildung 1: schematische Darstellung eines einseitig eingespannten Kragträgers unter Querkraftbelastung (a) Spannungstrajektorien im Kragträger (in Anlehnung an [9]) (b) Zug-Druckspannungsverteilung über den Querschnitt (c) Schubspannungsverteilung über den Querschnitt (d)

Ausgehend von den anisotropen Beanspruchungen ist die Berücksichtigung von anisotropen Elementarzellen und somit anisotropen Meso-Strukturen im Sinne einer beanspruchungsgerechten Gestaltung der Struktur sowie der gebotenen Möglichkeiten von AM zielführend. Um die Auslegung von Meso-Strukturen mit Blick auf Beanspruchungsgerechtheit zu systematisieren, müssen die Eigenschaften verschiedener Elementarzellen abhängig von ihren beeinflussbaren konstruktiven Parametern, wie $z$. B. äußere Abmessungen oder Strukturdicke der Elementarzellen, bestimmt werden. Diese Zusammenhänge werden über die simulativ ermittelten äquivalenten Eigenschaften bisher nicht ersichtlich, sodass eine direkte Auslegung der Meso-Strukturen darüber nicht möglich ist. Im Gegensatz hierzu wurden zur konventionellen Berechnung häufig verwendeter massiver Bauteile Theorien, z. B. die Balkentheorie, entwickelt, die eine anschauliche Berechnung mittels axiomatischer Modelle ermöglichen. Dabei unterstützt der konstruktive Bezug der Berechnungsparameter die Auslegung besser als moderne FEMSimulationsverfahren.

Daher werden in diesem Beitrag beispielhaft Stabgitter-Strukturen über die Analogie zu zweidimensionalen Stabwerken angenähert und mithilfe konventioneller Fachwerkstheorien berechnet. Damit wird ermittelt, welche Parameter für eine Auslegung Elementarzelleneigenschaften und somit Meso-Struktur-Eigenschaften relevant sind, auf 
welche Weise und in welchem Maß die Parameter die Eigenschaften der Meso-Struktur beeinflussen und wie die Eigenschaften in Abhängigkeit der Parameter der Elementarzelle beschrieben werden können. Im Zuge dessen wird folgende Forschungsfrage untersucht: Welche Modelle von additiv gefertigten Elementarzellen und Meso-Strukturen eignen sich zur Formulierung analytischer Berechnungsgleichungen für die zielgerichtete Bauteilauslegung?

\section{Lösungsansatz}

Im Gegensatz zur Berechnung von Bauteilen über äquivalente Kontinuumseigenschaften, werden zur konstruktiven Auslegung der Meso-Strukturen die relevanten konstruktiven Parameter der Elementarzelle über die Analogie zu 2D-Stabwerken abgeleitet.

\subsection{Modell und Vorgehensweise}

Zur Ermittlung der relevanten konstruktiven Einflussgrößen der Elementarzelle auf die Eigenschaften der Meso-Struktur werden als Modell 2D-Stabwerke unter mechanischen Belastungsfällen wie Zug bzw. Druck, Biegung und Querkraft, betrachtet, die analytisch berechenbar sind. Die 2D-Stabwerke ermöglichen durch ihre Ähnlichkeit zu Stabgittern, wie in Abbildung 2 erkennbar, eine Übertragbarkeit der Erkenntnisse auf 3D-Stabgitterzellen. Als Beispiel wird hier der einseitig eingespannte Kragträger näher betrachtet. Für die Berechnung wird angenommen, dass die Stabbedingungen gelten und eine freie Deformation auftritt, da analog in 3D-Meso-Strukturen ebenfalls oft Stabgeometrien vorliegen, die außerhalb der Knotenpunkte nicht in ihrer Deformation behindert werden. Dabei werden die Einflüsse der Parameter der Stabwerkzelle deutlich und ein Rückschluss auf relevante Auslegungsparameter bei Meso-Strukturen kann gezogen werden.

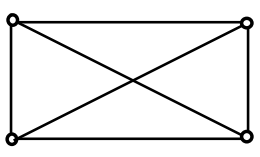

a) Stabwerkzelle

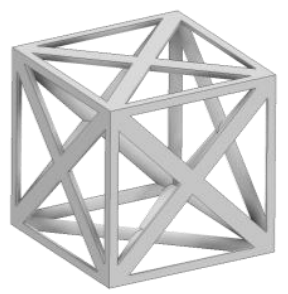

b) kubisch-flächenzentriert

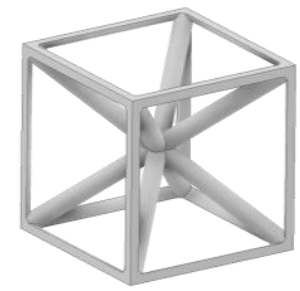

c) kubisch-raumzentriert

Abbildung 2: beispielhafte Stabwerkzelle (a) als vereinfachtes Modell für 3D-Meso-Strukturen, wie kubischflächenzentrierte (b) oder kubisch-raumzentrierte (c) Gitterstrukturen

\subsection{Relevante Einflussparameter}

Die Untersuchung der genannten Lastfälle für einzelne Stabwerkzellen und der Vergleich mit der analytischen Berechnung eines massiven Kragträgers ergibt die äquivalenten Eigenschaften für den jeweiligen Belastungsfall. Hier wird beispielhaft die Steifigkeit betrachtet, die auch in der Literatur vielfach als äquivalente Kontinuumsgröße untersucht wird [5-8]. Für den Kragträger unter Zug (bzw. analog für Druck), wie in Abbildung 3, wird das Verhältnis der Steifigkeiten von Werkstoff und Stabwerkzelle bestimmt, indem die Längung der Stabwerkzelle und eines massiven Balkenabschnitt mit gleichen Außenmaßen desselben Werkstoffs mit der Steifigkeit $E_{S}$, gleichgesetzt werden, also das gleiche Verhalten abgebildet wird. Löst man die Gleichung nach dem Verhältnis der Elastizitätsmoduln auf, erhält man Gleichung (1). (basierend auf [10])

$$
\frac{E_{\mathrm{S}}}{E^{*}}=\frac{b}{2 d} \frac{1}{1+\cos ^{3} \alpha} \quad \text { mit } \quad \cos \alpha=\frac{1}{\sqrt{1+\frac{b^{2}}{l^{2}}}}
$$


Anhand des Verhältnisses werden die folgenden relevanten Einflussgrößen deutlich: der Werkstoff, die relativen Dimensionen $b / 2 d$ der Stabwerkzellen-Stäbe mit der Strukturdicke $d$ und die Schlankheit $b / l$ der Stabwerkzelle. Der Werkstoff wird beschrieben durch seine linearelastischen Kennwerte, den Elastizitätsmodul bzw. übertragen auf andere Lastfälle den Schubmodul. Des Weiteren ist für komplexe Strukturen die Querkontraktionszahl ein relevanter Einflussparameter. Diese ist in Gleichung (1) nicht erkennbar, da die Berechnung unter der Annahme von Zug-/Druck-Stäben durchgeführt wurde, wobei die Querkontraktion vernachlässigt wird. Übertragen auf die Elementarzellen von 3D-Meso-Strukturen entspricht die Strukturdicke der Dicke von Stäben, Platten oder Schalen und zu Höhe und Breite kommt die Tiefe als dritte Dimension bei der Beschreibung der Elementarzellengröße und -schlankheit hinzu. Die Strukturdicke wird im Bereich der 3D-Meso-Strukturen oft über die relative Dichte beschrieben und auch die Elementarzellengröße wurde analog bei 3D-Meso-Strukturen als relevanter Parameter identifiziert [4, 11-13], was die Übertragbarkeit der identifizierten Einflussparameter auf 3D-Meso-Strukturen bestätigt. Die Schlankheit beschreibt die geometrische Anisotropie der Stabwerkzelle und entfällt bei isotropen Zellen. Zur beanspruchungsgerechten Auslegung kann eine anisotrope Zelle zweckmäßig sein. Für die mathematische Beschreibung der Anisotropie in der Meso-Struktur ist, wie bei anderen anisotropen Werkstoffen, z. B. Faser-Kunststoff-Verbunden, eine Tensorschreibweise der Steifigkeit erforderlich vgl. $[14,15]$.

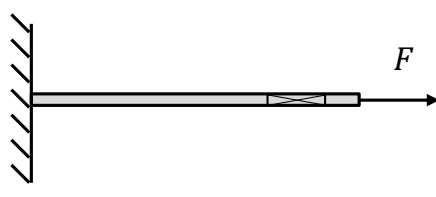

a) Kragträger

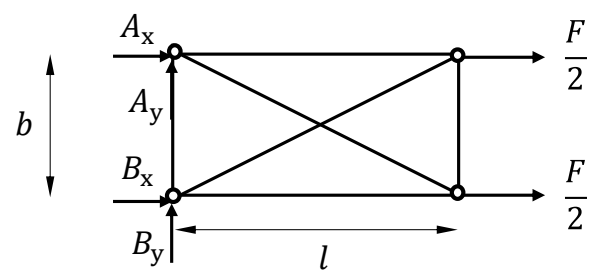

b) Stabwerkzelle

Abbildung 3: schematische Darstellung eines Kragträgers unter Zugbelastung (a) Stabwerkzelle unter Zugbelastung, die Kraft wird jeweils zur Hälfte an den Knotenpunkten angesetzt (b)

Bei der Integration von Meso-Strukturen in Bauteile wird von einem ein gestalteten Volumenbauteil ausgegangen, dessen Außenhaut und äußere Gestalt (weitestgehend) erhalten bleiben soll. Aus der Integration werden weitere auslegungsrelevante Einflüsse deutlich: Segmentierung und Orientierung. Die Segmentierung beschreibt, wie die ggf. variierenden Meso-Struktur-Zellen innerhalb des Bauteils verteilt sind und wie die Anzahl bzw. Größe relativ zum Bauteil gewählt ist. Die Orientierung definiert die Ausrichtung der MesoStruktur-Zellen innerhalb des Bauteils bzw. zur Bauteilgeometrie. Die Orientierung ist für die Berechnung der Meso-Struktur über eine Transformationsmatrix vom Meso-Struktur-ZellenKoordinatensystem (KOS) in das Bauteil-KOS möglich. Um eine beanspruchungsgerechte Auslegung der Meso-Struktur zu gewährleisten, sollten die Meso-Struktur-Zellen entlang der Hauptbeanspruchungen, für die sie gestaltet wurden, ausgerichtet sein. Das heißt, es wird jeweils „viel Material in Hauptspannungsrichtung orientiert“ vgl. [9]. Am Beispiel des Kragträgers unter Biegebelastung ist dies vereinfacht mit Stabwerkzellen in Abbildung 4 dargestellt. Dabei sind Stabwerkzellen mit jeweils unterschiedlicher Schlankheit vorgesehen, deren Orientierung und Verteilung gemäß der Beanspruchung gewählt ist. In den Randbereichen, in denen Längszug- bzw. -druckspannungen überwiegen, sind die Diagonalstäbe stärker in Richtung der Längsachse des Kragträgers ausgerichtet als im mittleren Bereich, in dem die Schubbeanspruchung überwiegt und die Diagonalstäbe stärker senkrecht zur Längsachse des Kragträgers ausgerichtet sind. Bei der Kombination unterschiedlicher Zellen, die in Form, Größe, Topologie oder Orientierung variieren, ist zu beachten, dass in den Kontaktbereichen zwischen den Zellen durch die 
Eigenschaftsänderung, z. B. einen Steifigkeitssprung, erhöhte Beanspruchungen auftreten können. Um ein Versagen (in Bereichen erhöhter Beanspruchungen) durch Eigenschaftsgradienten zu verhindern, sind Kriterien für die zulässigen Eigenschaftsunterschiede benachbarter Zellen zu definieren, mit denen sichergestellt werden kann, dass der Zellenverbund erhalten bleibt. Für das Beispiel des Balkens ist ein möglicher Ansatz für ein solches Kriterium die Zellen entsprechend der Annahmen auszulegen, die der konventionellen analytischen Berechnung homogener massiver Strukturen zugrunde liegen: Die üblichen Theorien gehen davon aus, dass die Querschnitte unter Biegeverformung eben bleiben.

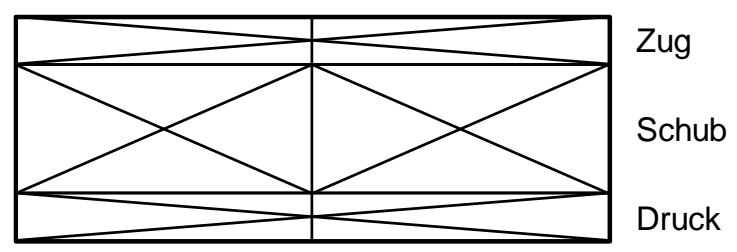

Abbildung 4: mögliche, beanspruchungsangenäherte Segmentierung, d. h. Variation und Verteilung, von Stabwerkzellen im Abschnitt eines Kragträgers unter Querkraftbelastung; für Zug und Druck in den Außenbereichen sind die Diagonalstäbe stärker in Längsrichtung ausgerichtet, für Schub im Zentrum stärker quer zum Kragträgerabschnitt

Aus dem Vergleich unterschiedlicher 2D-Stabwerkzellen und auf Basis von Erkenntnissen in der Literatur zu 3D-Meso-Struktur-Zellen wird außerdem deutlich, dass die Materialanordnung (auch als Topologie bezeichnet) innerhalb des Elementarzellen-Volumens, ein entscheidender Einflussparameter ist [4, 11]. Für 3D-Meso-Struktur-Zellen wurde der Einfluss der Materialanordnung gemeinsam mit den Geometrieanteilen der äquivalenten Eigenschaften bisher über Funktionen beschrieben, die für ausgewählte Zellen jeweils individuell aus Simulationen abgeleitet wurden [5]. Die ermittelten Einflussparameter sind relevant für die Bestimmung von äquivalenten Eigenschaften unterschiedlicher Elementarzellen ohne vorherige Simulation. Die Beschreibung abhängig von den Parametern ermöglicht überdies die Eigenschaften gemäß Anforderungen in Bauteilen einzustellen.

\subsection{Auswirkung des Einflusses auf die Steifigkeit}

Anhand der Steifigkeit der Stabwerkzellen bei Zug-, Biege- und Querkraftbelastung werden die Wirkungen der Einflussparameter auf die Steifigkeit analysiert. Dabei wird deutlich, dass die Auswirkungen vom Lastfall abhängig sind. Die Steifigkeit wird in allen Lastfällen durch den Einsatz eines Werkstoffs mit höherem Elastizitätsmodul des Werkstoffs erhöht. Ebenfalls steigert der Stabquerschnitt mit Breite $t$ und Höhe $d$ die Steifigkeit in den drei Beanspruchungsfällen linear. Die Länge $l$ und Breite $b$ der Stabwerkzelle gehen durch ihr Verhältnis beschreibbar durch den Winkel $\alpha$ ein. Mit steigendem Verhältnis $b / l$ steigt die Steifigkeit gegenüber Belastung durch Querkraft an und die Steifigkeit gegenüber Zugbelastung nimmt ab, da "wenig Material in Beanspruchungsrichtung orientiert ist“. Bei Zugbelastung wirkt sich das Verhältnis $b / l$ zunächst nicht auf die Steifigkeit aus, allerdings führt eine zunehmende Breite $b$ (und damit gleichzeitig der Länge $l$ ) zu einer Reduktion der Zug-/Drucksteifigkeit der Stabwerkzelle. Die Auswirkung der Einflüsse wurde anhand der analytisch hergeleiteten Absenkung bzw. Längung unter aufgebrachter Kraft untersucht. Zur Evaluierung der Ergebnisse wurden die Absenkung und die sich ergebende Steifigkeit für den jeweiligen Lastfall mit FEM-Simulationsergebnissen von dünnwandigen Rahmenmodellen mit gleichem Aufbau verglichen, die eine gute Übereinstimmung aufweisen. In Abbildung 5 ist der Vergleich für eine Stabwerkzelle unter Querkraft gezeigt.

Aus den unterschiedlichen Auswirkungen der Einflussparameter in den betrachteten Belastungsszenarien wird deutlich, dass die Eigenschaften von Meso-Strukturen 
beanspruchungsspezifisch bestimmt werden müssen. Somit können auch die aus Simulationen ermittelten äquivalenten Eigenschaften stets nur für den jeweils simulierten Lastfall für weitere Berechnungen verwendet werden. Dies entspricht dem Zusammenfassen von Elastizitätsmodul und Geometrie, etwa die Fläche für Zug- bzw. Druckspannungen oder Flächenträgheitsmomente im Fall von Biegespannungen, wie es bei der analytischen Berechnung von Bauteilen mit einem homogenen Werkstoff ebenfalls durchgeführt wird. Zur Verdeutlichung dieses Zusammenhangs wird vorgeschlagen auch die äquivalenten Kontinuumsgrößen, wie sie in Simulationen jeweils bestimmter Lastfälle und entsprechender Beanspruchungsfälle ermittelt werden, als zusammengesetzte Größen aus Werkstoff- und Geometrieeinfluss zu bezeichnen [16]. Für anisotrope Strukturen wird die Steifigkeit durch einen Tensor beschrieben, dessen Einträge dann jeweils den Steifigkeitswerten $k_{\mathrm{eq}}^{*}$ für die unterschiedlichen Richtungen entspricht.

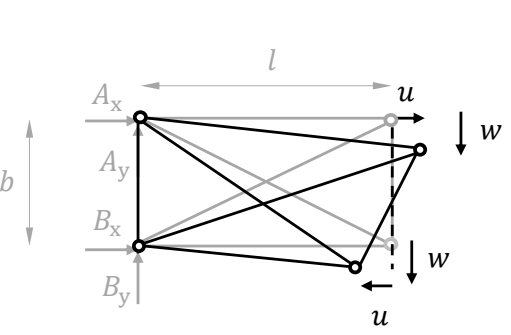

a) analytische Berechnung $u=0,003 \mathrm{~mm}$ $w=0,012 \mathrm{~mm}$
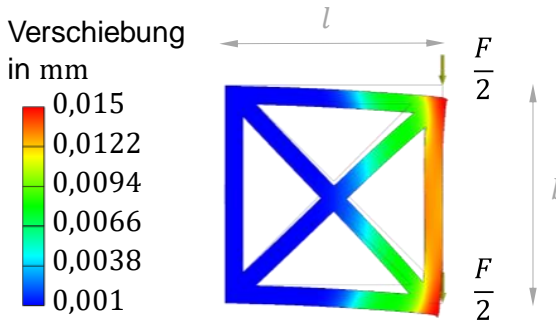

$F=100 \mathrm{~N}$

$b=l=27,5 \mathrm{~mm}$

$E=70000 \mathrm{~N} / \mathrm{mm}^{2}$

$v=0,3$

b) FEM-Simulation

$u=0,002 \mathrm{~mm}$

$w=0,012 \mathrm{~mm}$

Abbildung 5: Vergleich der Ergebnisse der analytischen Berechnung unter der Annahme kleiner Deformationen

(a) der Stabwerkzelle und FEM-Simulation des analogen Rahmenwerks (b)

\subsection{Berechnungsansatz für 2D-Zellenstrukturen}

Für die beanspruchungsgerechte Auslegung von Bauteilen mit Meso-Strukturen ist i. d. R. eine Segmentierung erforderlich, wobei unterschiedliche Elementarzellen beanspruchungsgemäß sind. Für einen 2D-Balken unter Querkraft beispielsweise nähert eine Segmentierung in schmale Randbereiche mit Stabwerkzellen, die eine Ausrichtung in Längsrichtung aufweisen und ein deutlich breiterer Bereich im Zentrum, in dem die Stabwerkzellen stärker in Querrichtung orientiert sind, eine beanspruchungsgerechte Eigenschaftsverteilung der Stabwerkzellen an, wie in 4.2 bereits erläutert. Eine solche Segmentierung ist in Abbildung 6 für einen Abschnitt eines Balkens unter Querkraft dargestellt.

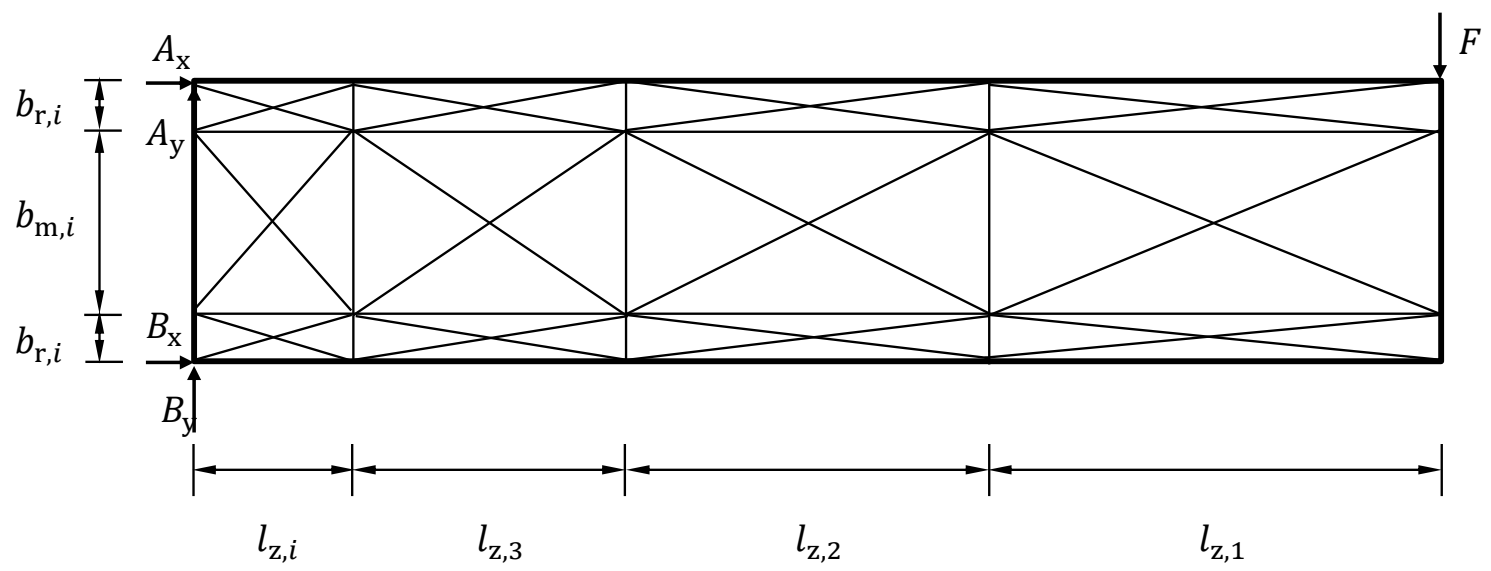

Abbildung 6: Abschnitt eines Balkens unter Querkraft, segmentiert in mehrere Stabwerkzellen gemäß Beanspruchung 
Um überhöhte Beanspruchungen zu vermeiden, wird als Bedingung angenommen, dass der Querschnitt des Balkens analog zu den Annahmen der analytischen Berechnungstheorien der Balkenbiegung, eben bleiben muss. Daraus ergibt sich, dass die Verformung am Übergang zwischen zwei Stabwerkszellen gleich ist, wie in Abbildung 7 b) dargestellt ist. Zur besseren Anschaulichkeit wurde ein Beispiel mit nur drei Schichten mit direkt nachvollziehbaren Beanspruchungs- und Verformungseigenschaften gewählt. Die Segmentierung wurde so gewählt, dass vereinfachend angenommen werden kann, dass die Querkraft $F_{\mathrm{Q}}$ über die Stabwerkzellen der mittleren Schicht übertragen wird und die Stabwerkzellen im Randbereich hauptsächlich Längszug- bzw. -druckkräfte $F_{\mathrm{M} / 2}$ übertragen. Aufgrund der geringen Größe von Meso-Struktur-Zellen können die Kräfte über die Elementarzellenbreite im Allgemeinen als konstant angenommen werden. Die Zug- bzw. Druckkräfte werden jeweils im Kontaktbereich zwischen den Stabwerkzellensegmenten übertragen, wie in Abbildung 7 a) gezeigt wird. Aufgrund der Bedingung am Übergang zwischen den Stabwerkzellen, ist die Verschiebung hier gleichzusetzen. Diese Bedingung kann basierend auf den Verhältnissen der Zellensteifigkeiten zu den entsprechenden Kräften analytisch formuliert werden (siehe Abbildung $7 \mathrm{c}$ ). Das Verhältnis der Zug-Druck-Zellensteifigkeit der Randschichtzellen $K_{\mathrm{ZD}, \mathrm{r}} \mathrm{zu}$ den Längskräften $F_{\mathrm{M}}$, die dem Biegemoment im Balkenquerschnit entsprechen, wird gleichgesetzt mit Verhältnis der Verdrehzellensteifigkeit $K_{\Phi, m}$ der mittleren Zellenschicht zur Querkraft.

a)

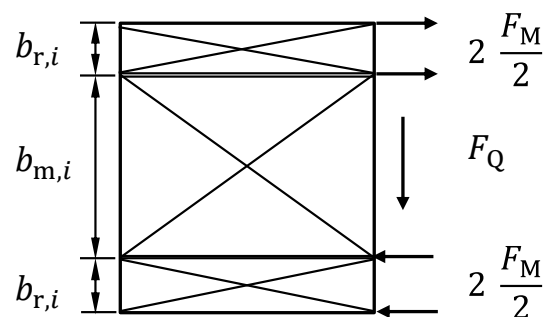

b)

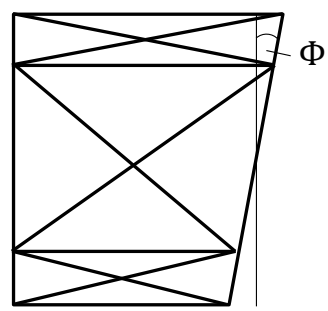

$$
\begin{gathered}
\frac{K_{\Phi, \mathrm{m}} \frac{b_{\mathrm{m}}+b_{\mathrm{r}}}{2}}{F_{\mathrm{Q}}}=\frac{K_{\mathrm{ZD}, \mathrm{r}}}{F_{\mathrm{M}}} \\
\text { c) } \frac{K_{\mathrm{ZD}, \mathrm{r}}}{K_{\Phi, \mathrm{m}}}=\frac{F_{\mathrm{M}}}{F_{\mathrm{Q}}} \frac{b_{\mathrm{m}}+b_{\mathrm{r}}}{2}
\end{gathered}
$$

Abbildung 7: Kraftaufteilung auf die Stabwerkzellen für die Berechnung (a) Verschiebung der Stabwerkzellen unter Ebenbleiben des Querschnitts (b) Verhältnis der Zellensteifigkeiten bedingt durch den ebenen Querschnitt

Zur besseren Veranschaulichung werden hier im Beispiel weitere vereinfachende Annahmen getroffen. Es wird vorausgesetzt, dass die Randschicht sehr viel schmaler ist als die mittlere Schicht $\left(b_{\mathrm{m}} \gg b_{\mathrm{r}}\right)$ und dass somit die Verdrehsteifigkeit des Randbereichs deutlich geringer ist als in der mittleren Schicht $\left(K_{\Phi, \mathrm{m}} \gg K_{\Phi, \mathrm{r}}\right)$. Des Weiteren wird vereinfachend angenommen, dass die Stabwerkzellen in der mittleren Schicht annähernd quadratisch sind und die Länge $l_{\mathrm{z} i}$, und die Breiten $b_{\mathrm{m} i}$ und $b_{\mathrm{r} i}$ der Stabwerkzellen am Rand und in der Mitte des Balkens sowie die Dicke der Stäbe in den mittleren Stabwerkzellen $d_{\mathrm{m} i}$ konstant sind. Unter diesen Annahmen kann für eine beanspruchungsgerechte Gradierung der Stabwerkzellen im Randbereich über die Stabdicke die geometrische Bedingung abhängig von der Zellenposition abgeleitet werden:

$$
d_{\mathrm{r} i}=i d_{\mathrm{m}}\left(\frac{b_{\mathrm{m}}}{b_{\mathrm{m}}+b_{\mathrm{r}}}\right)^{2} \frac{1}{1+\cos ^{2}\left(\alpha_{\mathrm{r}}\right)} \quad \text { mit } \quad \frac{K_{\mathrm{ZD}, \mathrm{r}}}{K_{\Phi, \mathrm{m}}}=\frac{4 l_{\mathrm{z}} i}{\left(b_{\mathrm{m}}+b_{\mathrm{r}}\right)^{2}}
$$

\subsection{Anwendbarkeit der äquivalenten Eigenschaften in analytischer Berechnung}

Anhand der geometrischen Bedingung, die sich aus der Ebenheit des Querschnitts ergibt, wird deutlich, dass das Verhalten der Stabwerkzellen den Annahmen analytischer Berechnungsvorschriften, wie z. B. dem Bernoulli- oder Timoschenko-Balken, nicht zwingend genügt. Der Vergleich wurde im Zweidimensionalen durchgeführt, um die Bedingungen einfach prüfbar zu halten. 3D-Meso-Strukturen weisen jedoch dieselben Charakteristika auf, 
d. h. Verformungen treten lokal in den Bereichen auf, wo Material in den Elementarzellen vorliegt und entsprechend des Deformationsverhaltens der typischen Strukturen, wie Stäbe, Platten oder Schalen. Ein daraus gebildeter Querschnitt, der den deformierten Rand einer Elementarzelle abbildet, ist nur in Ausnahmefällen eben und/oder senkrecht zur Mittelebene der Elementarzelle. Die Übertragbarkeit der Annahmen und somit die Anwendung der analytischen Berechnungsvorschriften auf Stabwerkzellen und in ähnlicher Form auch 3DMeso-Strukturen ist nur dann möglich, wenn die Zellen, analog zum Beispiel des segmentierten Stabwerkbalkens im Vorhinein auf ebenjene Annahmen ausgelegt worden sind.

\section{Fazit und Ausblick}

Eine aufwendige iterative Auslegung von Meso-Strukturen durch wiederholte Simulationen kann vermieden und ihre Potentiale gezielt und umfänglich genutzt werden, wenn die Gestaltung der inneren Bereiche von Volumenbauteilen methodisch unterstützt beanspruchungsgerecht ausgelegt werden können. Voraussetzung hierfür ist, dass die (konstruktiven) Einflussfaktoren auf die Eigenschaften von Meso-Strukturen bzw. ihren Elementarzellen bekannt sind.

In diesem Beitrag wurden ausgehend von zweidimensionalen Stabwerkstrukturen, die aufgrund der gezielten Masseanordnung im Stabwerk als hinreichend ähnliches, aber deutlich anschaulicheres Modell für 3D-Meso-Strukturen dienen, verwendet und Einflüsse auf deren Eigenschaften identifiziert, die sich auf 3D-Meso-Strukturen übertragen lassen. Für eine beanspruchungsgerechte Integration von Meso-Strukturen in Bauteilen ist besonders die Betrachtung anisotroper Elementarzellen relevant, deren Eigenschaften durch ihre Form, Topologie, äußeren Abmessungen, Abmessungen der inneren Strukturelemente, Schlankheit und Orientierung festgelegt sind. Die Schlankheit anisotroper Zellen im Bereich der Stabwerke beeinflusst die Eigenschaften (z. B. die Steifigkeit) der Elementarzelle abhängig von der Beanspruchung unterschiedlich. Ein weiterer Einfluss auf die Eigenschaften der Elementarzellen ist deren Materialanordnung bzw. Topologie innerhalb des Elementarzellenvolumens, die durch die Wahl einer Elementarzelle festgelegt wird.

Der Ansatz zur Entwicklung einer Auslegungsmethode wird anhand des Beispiel von 2D-Stabwerkszellen und eines Biegebalkens abgeleitet, erläutert und dessen prinzipielle Eignung abgesichert. Ausgehend von den identifizierten Einflussparametern wird in weiterer Forschung der Übergang von der Beschreibung von 2D-Stabwerken auf die auslegungsgerechte Beschreibung der Eigenschaften von 3D-Meso-Strukturen unter Berücksichtigung verschiedener Zelltypen und komplexer Strukturen erarbeitet.

Des Weiteren wird angestrebt die Auslegung von Bauteilen mit Meso-Strukturen zu operationalisieren, wobei folgende Arbeiten unterstützt werden. Ausgehend von einer Analyse der Beanspruchungen im Volumenbauteil, z. B. mittels FEM des massiven Bauteils, werden charakteristische Beanspruchungsbereiche im Bauteil identifiziert. Für diese werden jeweils geeignete Elementarzellentopologien gewählt und die Parameter der Elementarzellen, im Wesentlichen Orientierung, Schlankheit, äußere Abmessungen und Abmessungen der inneren Strukturelemente, entsprechend der Beanspruchung gewählt bzw. dimensioniert. Hierfür werden ausgehend von den hier vorgestellten Ansätzen Vorgehensweisen und Hilfsmittel erarbeitet, z. B. zur Bestimmung diskretisierter Steifigkeitstensoren basierend auf den Eigenschaften der Elementarzellen der Meso-Strukturen. Mithilfe dieser Eigenschaftstensoren können dann die Bauteileigenschaften über vereinfachte Berechnungen zunächst näherungsweise ermittelt werden, um die Eignung der Gestaltung der Meso-Struktur zu überprüfen und grundsätzlich zu optimieren. Ebenfalls ist die Fertigbarkeit der Struktur zu untersuchen und ggf. die Topologieauswahl, die Dimensionierung der Elementarzelle sowie die Ausführung der Meso-Struktur anzupassen.

Ein weiterer Vorteil der so definierten Elementarzellen besteht in der einfachen Kombinierbarkeit der gezielten, parameterbasierten Auslegung mit geringem Aufwand und der 
exakten Nachweisrechnung des Bauteils mit üblichen FEM-Simulationsrechnungen. Es besteht die Möglichkeit, aus den parametrisch beschriebenen Eigenschaften der Elementarzellen und Meso-Strukturen bereichsweise äquivalente Kontinuumseigenschaften abzuleiten. Damit kann das Bauteil bei der FEM-Nachweisrechnung unabhängig von der ausgelegten Meso-Struktur als Kontinuum modelliert und simuliert werden.

\section{Literaturverzeichnis}

[1] Nguyen, Jason ; Park, Sang-In ; Rosen, David: Heuristic optimization method for cellular structure design of light weight components. In: International Journal of Precision Engineering and Manufacturing 14 (2013), Nr. 6, S. 1071-1078

[2] Bici, Michele ; Brischetto, Salvatore ; Campana, Francesca ; Ferro, Carlo Giovanni ; Seclì, Carlo ; Varetti, Sara ; Maggiore, Paolo ; Mazza, Andrea: Development of a multifunctional panel for aerospace use through SLM additive manufacturing. In: Procedia CIRP 67 (2018), S. 215-220

[3] Caiazzo, Fabrizia ; Alfieri, Vittorio ; Bujazha, Brahim David: Additive manufacturing of biomorphic scaffolds for bone tissue engineering. In: The International Journal of Advanced Manufacturing Technology 113 (2021), 9-10, S. 2909-2923

[4] Tancogne-Dejean, Thomas ; Diamantopoulou, Marianna ; Gorji, Maysam B. ; Bonatti, Colin ; Mohr, Dirk: 3D Plate-Lattices: An Emerging Class of Low-Density Metamaterial Exhibiting Optimal Isotropic Stiffness. In: Advanced materials (Deerfield Beach, Fla.) 30 (2018), Nr. 45, e1803334

[5] Vega-Moreno, Afrodisio ; Tenegi Sanginés, Fabio ; Márquez-Rodríguez, Juan Francisco ; Calvo-Tovar, Juan; Schnetler, Hermine ; Atkins, Carolyn ; Miller, Chris ; Morris, Katherine ; Snell, Robert M. ; van de Vorst, Bart; Dufils, Joris ; Brouwers, Leon ; Roulet, Mélanie ; Hugot, Emmanuel ; Farkas, Szigfrid ; Mezo, Gyorgy: Design for additive manufacture (DfAM): the "equivalent continuum material" for cellular structures analysis. In: Angeli, George Z.; Dierickx, Philippe (Hrsg.): Modeling, Systems Engineering, and Project Management for Astronomy IX : SPIE, 2020 - 2020, S. 84

[6] Al-Wattar, Tahseen Abdulridha Ali: Developing equivalent solid model for lattice cell structures using numerical approaches. Dayton, wright state university. Dissertation. 2020

[7] Alwattar, Tahseen A. ; Mian, Ahsan: Developing an Equivalent Solid Material Model for BCC Lattice Cell Structures Involving Vertical and Horizontal Struts. In: Journal of Composites Science 4 (2020), Nr. 2, S. 74

[8] Souza, José ; Großmann, Alexander ; Mittelstedt, Christian: Micromechanical analysis of the effective properties of lattice structures in additive manufacturing. In: Additive Manufacturing 23 (2018), Nr. 2, S. 5369

[9] Teufelhart, Stefan: Belastungsoptimiertes Design von Gitterstrukturen für die additive Fertigung nach dem bionischen Prinzip der kraftflussgerechten Gestaltung. Fakultät für Maschinenwesen. 2016

[10] Gross, Dietmar ; Hauger, Werner ; Schröder, Jörg ; Wall, Wolfgang A.: Technische Mechanik 2. Berlin Heidelberg : Springer Berlin Heidelberg, 2021

[11] Tancogne-Dejean, Thomas ; Mohr, Dirk: Elastically-isotropic truss lattice materials of reduced plastic anisotropy. In: International Journal of Solids and Structures 138 (2018), S. 24-39

[12] Catchpole-Smith, S. ; Sélo, R.R.J. ; Davis, A. W. ; Ashcroft, I. A. ; Tuck, C. J. ; Clare, A.: Thermal conductivity of TPMS lattice structures manufactured via laser powder bed fusion. In: Additive Manufacturing 30 (2019), S. 100846 .

[13] Merkt, Simon: Qualifizierung von generativ gefertigten Gitterstrukturen für maßgeschneiderte Bauteilfunktionen. Aachen, RWTH Aachen, Institut für Maschinenwesen. Dissertation. 2015.

[14] Schürmann, Helmut: Konstruieren mit Faser-Kunststoff-Verbunden. 2., bearbeitete und erweiterte Auflage. Berlin, Heidelberg : Springer-Verlag Berlin Heidelberg, 2007 (VDI-Buch)

[15] Gross, Dietmar ; Hauger, Werner ; Wriggers, Peter: Technische Mechanik 4. Berlin, Heidelberg : Springer Berlin Heidelberg, 2018

[16] Öchsner, Andreas: Theorie der Balkenbiegung. Wiesbaden : Springer Fachmedien Wiesbaden, 2016 